SUBJECT AREAS:

ELECTRONIC STRUCTURE

TWO-DIMENSIONAL MATERIALS

Received

5 December 2013

Accepted

31 January 2014

Published

27 February 2014

Correspondence and requests for materials should be addressed to H.T. (terroh@rpi.edu)

* Current address: Department of Physics, Applied Physics and Astronomy, Rensselaer Polytechnic Institute, IC25 Johnson-Rowland Science Center, 110 Eighth Street, Troy, NY 12180 , USA.

\section{New First Order Raman-active Modes in Few Layered Transition Metal Dichalcogenides}

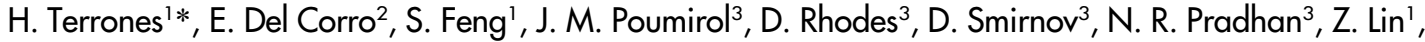 \\ M. A. T. Nguyen ${ }^{4}$, A. L. Elías' ${ }^{1}$, T. E. Mallouk ${ }^{1,4}$, L. Balicas ${ }^{3}$, M. A. Pimenta ${ }^{2} \&$ M. Terrones ${ }^{1,4,5}$
}

\begin{abstract}
'Department of Physics and Center for 2-Dimensional and Layered Materials, The Pennsylvania State University, 104 Davey Lab, University Park, PA 16802, USA, ${ }^{2}$ Universidade Federal de Minas Gerais, Departamento de Física, Av. Antônio Carlos, 6627 Caixa Postal 702 - cep 30.123-970 Belo Horizonte/MG - Brazil, ${ }^{3}$ National High Magnetic Field Laboratory, Florida State University, Tallahassee-FL 323 10, USA, ${ }^{4}$ Department of Chemistry, The Pennsylvania State University, University Park, PA 16802, United States, ${ }^{5}$ Department of Materials Science and Engineering, The Pennsylvania State University, University Park, PA 16802, USA \& Research Center for Exotic Nanocarbons (JST), Shinshu University, Wakasato 4-17-1, Nagano, 380-8553, Japan.
\end{abstract}

Although the main Raman features of semiconducting transition metal dichalcogenides are well known for the monolayer and bulk, there are important differences exhibited by few layered systems which have not been fully addressed. WSe $e_{2}$ samples were synthesized and ab-initio calculations carried out. We calculated phonon dispersions and Raman-active modes in layered systems: $\mathrm{WSe}_{2}, \mathrm{MoSe}_{2}, \mathrm{WS}_{2}$ and $\mathrm{MoS}_{2}$ ranging from monolayers to five-layers and the bulk. First, we confirmed that as the number of layers increase, the $E^{\prime}, E^{\prime \prime}$ and $E_{2 g}$ modes shift to lower frequencies, and the $A^{\prime}{ }_{1}$ and $A_{1 g}$ modes shift to higher frequencies. Second, new high frequency first order $\mathrm{A}^{\prime}{ }_{1}$ and $\mathrm{A}_{1 \mathrm{~g}}$ modes appear, explaining recently reported experimental data for $\mathrm{WSe}_{2}, \mathrm{MoSe}_{2}$ and $\mathrm{MoS}_{2}$. Third, splitting of modes around $\mathrm{A}_{1}^{\prime}$ and $\mathrm{A}_{1 \mathrm{~g}}$ is found which explains those observed in $\mathrm{MoSe}_{2}$. Finally, exterior and interior layers possess different vibrational frequencies. Therefore, it is now possible to precisely identify few-layered STMD.

. he field of 2-Dimensional semiconducting transition metal dichalcogenides (STMDs) is growing very fast mainly due to: a) their moderate band gap which can be used for fabricating electronic devices such as field effect transistors $(\mathrm{FETs})^{1-7}$; b) the presence of a direct band gap in monolayered STMDs, ideal for optoelectronic devices ${ }^{8-11}$, and c) their unique valley polarization properties ${ }^{12-14}$. The direct band gap of monolayered SMTDs has been measured and it results in an intense photoluminescence (PL) signal ${ }^{9,11}$ which can be enhanced at the edges possibly due to edge passivation ${ }^{15}$. The non-destructive technique of Raman spectroscopy has been intensively used to characterize monolayered STMDs, and other than the first-order modes, double resonant and triple resonant processes have been recently identified ${ }^{16,17}$. Recently, important efforts in understanding the Raman spectroscopy of few layered systems have been carried out in $\mathrm{WSe}_{2}{ }^{18,19}$. However, a careful, systematic and deep study of the different Raman modes in few layered STMDs (FLSTMD) is needed since previous works use either the monolayered or the bulk systems to explain Raman spectra of FLSTMDs. Unfortunately, this kind of analysis might lead to an incorrect assignment of the active Raman modes ${ }^{20}$ since FLSTMDs exhibit different symmetries depending on the number of layers. In this manuscript, using first principles density functional perturbation theory (DFPT), we shed light on this subject and find that the particular symmetry in FLSTMD (e.g. $\mathrm{MoS}_{2}, \mathrm{WS}_{2}, \mathrm{WSe}_{2}, \mathrm{MoSe}_{2}$ ) results in new Raman-active modes that are neither present in the monolayer nor in the bulk spectra. In particular, we predict the existence of a new out-of-plane Raman-active mode, which is expected to be intense in the spectra of FLSTMDs such as $\mathrm{WSe}_{2}, \mathrm{MoSe}_{2}$ and $\mathrm{MoS}_{2}$. This mode has already been experimentally reported ${ }^{21-23}$, but neither fully understood nor studied in detail in different FLSTMDs. We present here a polarized Raman study of this new mode, on synthesized chemical vapor transport samples ${ }^{24-26}$, which supports our prediction. In our calculations, we additionally found a splitting in all Raman-active modes which explains the recently observed double and triple modes in $\mathrm{MoSe}_{2}$ when $\mathrm{L}=3, \mathrm{~L}=4$ and $\mathrm{L}=5$ for the $\mathrm{A}^{\prime}{ }_{1}$ and $\mathrm{A}_{\mathrm{g}}^{1}{ }^{22}$ (see table 1), in addition to other modes related to in-plane vibrations that differentiate between exterior (surface) and interior (bulk) layer vibrations.

Bulk trigonal prismatic STMDs belong to $\mathrm{P}_{3} / \mathrm{mmc}\left(\mathrm{D}_{6 \mathrm{~h}}{ }^{4}\right)$, which is a nonsymmorphic space group, and thus, in order to analyze their vibrations in the unit cell, we need to consider that their unit cell exhibits $\mathrm{D}_{6 \mathrm{~h}}$ point group 
Table 1 | First order Raman-active modes in $\mathrm{cm}^{-1}$ of few layered $\mathrm{WSe}_{2}, \mathrm{MoSe}_{2}, \mathrm{MoS}_{2}$ and WS (from L $=1$ to $\mathrm{L}=5$ layers) and the bulk, calculated using DFPT, and experimental results published in the literature (in yellow). The point symmetry of the unit cell is indicated in the first row by its Shöenflies symbol. An asterisk * indicates the more intense calculated signals, "ir" indicates that the mode is also infraredactive, "in" indicates that just the interior layers vibrate and "ex" represents that just the exterior layers vibrate. The lower frequency modes have not been included since some of them do not appear in the most common Raman configurations

\begin{tabular}{|c|c|c|c|c|c|c|}
\hline Structure & $\mathrm{L}=1\left(\mathrm{~cm}^{-1}\right) \mathrm{D}_{3 \mathrm{~h}}$ & $\mathrm{~L}=2\left(\mathrm{~cm}^{-1}\right) \mathrm{D}_{3 \mathrm{~d}}$ & $\mathrm{~L}=3\left(\mathrm{~cm}^{-1}\right) \mathrm{D}_{3 \mathrm{~h}}$ & $\mathrm{~L}=4\left(\mathrm{~cm}^{-1}\right) \mathrm{D}_{3 \mathrm{~d}}$ & $\mathrm{~L}=5\left(\mathrm{~cm}^{-1}\right) \mathrm{D}_{3 \mathrm{~h}}$ & Bulk $\left(\mathrm{cm}^{-1}\right) D_{6 h}$ \\
\hline $\mathrm{WSe}_{2}$ & $\begin{array}{l}175.75\left(\mathrm{E}^{\prime \prime}\right) \\
249.36\left(\mathrm{E}^{\prime}\right)^{* \mathrm{ir}} \\
250.23\left(\mathrm{~A}^{\prime}{ }_{1}{ }^{*}\right) \\
249.5\left(\mathrm{E}^{\prime}\right)^{21} \\
249.5\left(\mathrm{~A}^{\prime}{ }_{1}\right)^{21}\end{array}$ & $\begin{array}{l}176.20\left(\mathrm{E}_{\mathrm{g}}{ }^{2}\right)^{1} \\
176.26\left(\mathrm{E}_{\mathrm{g}}{ }^{1}\right)^{19} \\
\left.248.52\left(\mathrm{E}_{\mathrm{g}}\right)^{1}\right)^{*} \\
250.83\left(\mathrm{~A}_{\mathrm{lg}}\right)^{*} \\
306.97\left(\mathrm{~A}_{1 \mathrm{~g}}{ }^{2}\right)^{*} \\
309.00\left(\mathrm{~A}_{\mathrm{lg}}{ }^{2}\right)^{22} \\
310.00\left(\mathrm{~A}_{1 \mathrm{~g}}{ }^{2}\right)^{21}\end{array}$ & $\begin{array}{l}175.01\left(\mathrm{E}^{\prime \prime 2}\right) \\
175.58\left(\mathrm{E}^{\prime 2}\right)^{\mathrm{ir}} \\
175.84\left(\mathrm{E}^{\prime \prime 2}\right)^{19} \\
176.33\left(\mathrm{E}^{\prime \prime 2}\right)^{19} \\
247.83\left(\mathrm{E}^{\prime}\right)^{* i \mathrm{r}, \mathrm{ln}} \\
248.32\left(\mathrm{E}^{\prime}\right)^{* i r, \mathrm{Ex}} \\
248.31\left(\mathrm{E}^{\prime \prime \prime}\right)^{* \mathrm{Ex}} \\
249.16\left(\mathrm{~A}^{\prime},{ }_{1}\right) \\
251.23\left(\mathrm{~A}^{\prime}{ }^{\prime}\right)^{*} \\
306.57\left(\mathrm{~A}^{\prime}{ }^{\prime}{ }^{2}\right)^{*} \\
309.00\left(\mathrm{~A}^{\prime}{ }^{2}\right)^{22} \\
310.00\left(\mathrm{~A}^{\prime}{ }_{1}{ }^{2}\right)^{21}\end{array}$ & 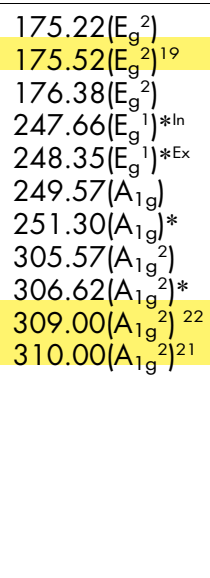 & 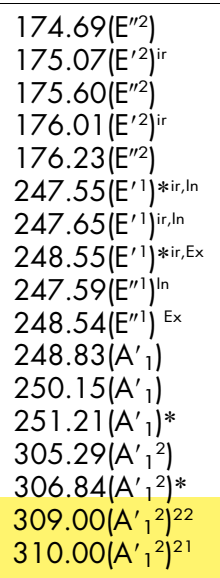 & $\begin{array}{c}175.01\left(E_{1 g}\right) \\
177.5\left(E_{1 g}\right)^{46} \\
247.78\left(E_{2 g}\right)^{*} \\
251.61\left(A_{1 g}\right)^{*} \\
248.0\left(E_{2 g}\right)^{22} \\
250.8\left(A_{1 g}\right)^{22} \\
247.00\left(E_{2 g}\right)^{30} \\
251.00\left(A_{1 g}\right)^{30} \\
250\left(E_{2 g}\right)^{46} \\
253\left(A_{1 g}\right)^{46}\end{array}$ \\
\hline $\mathrm{MoS}_{2}$ & $\begin{array}{c}284.00\left(E^{\prime \prime}\right) \\
383.61\left(E^{\prime}\right)^{* i r} \\
384.7\left(E^{\prime}\right)^{34} \\
384.0\left(E^{\prime}\right)^{29} \\
402.65\left(A^{\prime}{ }_{1}\right)^{*} \\
406.1\left(A^{\prime}{ }_{1}\right)^{34} \\
403.00\left(A^{\prime}{ }_{1}\right)^{29}\end{array}$ & $\begin{array}{l}283.78\left(\mathrm{E}_{\mathrm{g}}{ }^{2}\right)^{2} \\
\left.381.65\left(\mathrm{E}_{\mathrm{g}}\right)^{1}\right)^{*} \\
402.86\left(\mathrm{~A}_{1 \mathrm{~g}}\right)^{*} \\
463.84\left(\mathrm{~A}_{\mathrm{lg}_{\mathrm{g}}}\right)^{2}\end{array}$ & 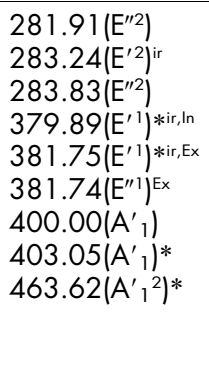 & $\begin{array}{l}281.79\left(\mathrm{E}_{\mathrm{g}}{ }^{2}\right) \\
283.28\left(\mathrm{E}_{\mathrm{g}}{ }^{2}\right)^{2} \\
379.57\left(\mathrm{E}_{\mathrm{g}}\right)^{* \ln } \\
381.54\left(\mathrm{E}_{\mathrm{g}}\right)^{* \mathrm{Ex}} \\
400.67\left(\mathrm{~A}_{\mathrm{lg}}\right) \\
402.80\left(\mathrm{~A}_{1 \mathrm{~g}}\right)^{*} \\
461.12\left(\mathrm{~A}_{1 \mathrm{~g}}{ }^{2}\right)^{2} \\
463.39\left(\mathrm{~A}_{l_{\mathrm{g}}}\right)^{*}\end{array}$ & 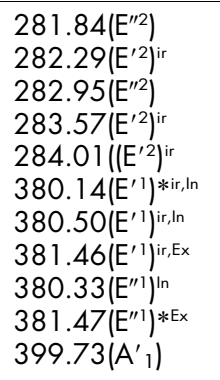 & $\begin{array}{c}282.05\left(\mathrm{E}_{1 \mathrm{~g}}\right) \\
286.00\left(\mathrm{E}_{1 \mathrm{l}}\right)^{47} \\
287.00\left(\mathrm{E}_{1 \mathrm{~g}}\right)^{48} \\
380.35\left(\mathrm{E}_{2 \mathrm{~g}}\right)^{*} \\
383.00\left(\mathrm{E}_{2 \mathrm{~g}}\right)^{48} \\
383.50\left(\mathrm{E}_{2 \mathrm{~g}}\right)^{22} \\
383.7\left(\mathrm{E}_{2 \mathrm{~g}}\right)^{34} \\
405.02\left(\mathrm{~A}_{1 \mathrm{~g}}\right)^{*} \\
408.6\left(\mathrm{~A}_{1 \mathrm{~g}}\right)^{22} \\
408.7\left(\mathrm{~A}_{1 \mathrm{~g}}\right)^{34}\end{array}$ \\
\hline
\end{tabular}


Table 1 | Continued

\begin{tabular}{|c|c|c|c|c|c|c|}
\hline Structure & $\mathrm{L}=1\left(\mathrm{~cm}^{-1}\right) \mathrm{D}_{3 \mathrm{~h}}$ & $\mathrm{~L}=2\left(\mathrm{~cm}^{-1}\right) \mathrm{D}_{3 \mathrm{~d}}$ & $\mathrm{~L}=3\left(\mathrm{~cm}^{-1}\right) \mathrm{D}_{3 \mathrm{~h}}$ & $\mathrm{~L}=4\left(\mathrm{~cm}^{-1}\right) \mathrm{D}_{3 \mathrm{~d}}$ & $\mathrm{~L}=5\left(\mathrm{~cm}^{-1}\right) \mathrm{D}_{3 \mathrm{~h}}$ & Bulk $\left(\mathrm{cm}^{-1}\right) D_{6 h}$ \\
\hline & & & & & $\begin{array}{l}401.43\left(\mathrm{~A}^{\prime}{ }_{1}\right) \\
403.44\left(\mathrm{~A}^{\prime}{ }_{1}\right)^{*} \\
461.28\left(\mathrm{~A}^{\prime}{ }_{1}{ }^{2}\right) \\
463.18\left(\mathrm{~A}^{\prime}{ }_{1}{ }^{2}\right)^{*} \\
463.00\left(\mathrm{~A}^{\prime}{ }_{1}{ }^{2}\right)^{23}\end{array}$ & \\
\hline
\end{tabular}

symmetry; this is not the case for the monolayered or FLSTMDs, which are considered quasi $2-D$ systems in the " $Z$ " direction. The monolayer in STMDs is non-centrosymmetric (no inversion symmetry) and its vibrational modes belong to the $\mathrm{D}_{3 \mathrm{~h}}$ point group. Therefore, in agreement with the irreducible representations of this point group, one must use the symbols $\mathrm{A}^{\prime}{ }_{1}$ and $\mathrm{E}^{\prime}$, for the out of plane and in-plane vibrations, respectively. For understanding the case of FLSTMDs $(\mathrm{L}=2,3,4,5 \ldots)$, their particular symmetries need to be analyzed since the symmetry changes when varying the number of layers from even to odd numbers. Systems with an odd number of layers $(\mathrm{L}=1,3,5, \ldots)$ exhibit $\mathrm{D}_{3 \mathrm{~h}}$ point-group symmetry, do not possess a center of inversion, and the out-of-plane Raman-active modes belong to the $\mathrm{A}^{\prime}{ }_{1}$ irreducible representation. For systems with an even number of layers $(L=2,4, \ldots)$, the modes belong to the $D_{3 d}$ point group which exhibits a center of inversion, and the out-ofplane Raman-active modes possess the $\mathrm{A}_{1 \mathrm{~g}}$ irreducible representation. In this context, it is important to emphasize that the absence of inversion symmetry plays a crucial role in nonlinear optical properties such as the second harmonic generation ${ }^{27,28}$, and valley polarization $^{12-14}$.

\section{Results}

We synthesized crystals of the trigonal prismatic phase of $\mathrm{WSe}_{2}$ by chemical vapor transport techniques ${ }^{24-26}$ (see methods section), and by adhesive tape exfoliation we were able to produce few layered $\mathrm{WSe}_{2}$ (See figure 1). Raman spectra were attained using four different laser lines (488 nm, $514.5 \mathrm{~nm}, 633 \mathrm{~nm}$ and $647 \mathrm{~nm}$ ) to study their vibrational properties depending on the number of layers (See figure $2 \mathrm{a}-2 \mathrm{~d}$ ), and photoluminescence studies were carried out to confirm the presence of the direct optical band gap at around $750 \mathrm{~nm}$ $(1.65 \mathrm{eV})$ for the monolayer ${ }^{22}$. It is noticed that as the number of layers increases, the PL intensity decreases, appearing two signals which shift to higher wavelengths (lower energies) agreeing with the work of Tonndorf et $\mathrm{al}^{22}$ (see figure $2 \mathrm{e}$ ).

We first considered the case of monolayered $\mathrm{WSe}_{2}$, in which the experimental Raman spectrum exhibits the presence of the perpendicular mode $\mathrm{A}^{\prime}{ }_{1}$ and the in-plane $\mathrm{E}^{\prime}$ almost degenerate at around $250 \mathrm{~cm}^{-119,21,22}$; according to our calculations $\mathrm{A}^{\prime}{ }_{1}$ is at $250.23 \mathrm{~cm}^{-1}$ and the $\mathrm{E}^{\prime}$ at $249.36 \mathrm{~cm}^{-1}$ (see table 1). By adding layers, we observed that the out of plane modes $\mathrm{A}^{\prime}{ }_{1}\left(\mathrm{~A}_{1 \mathrm{~g}}\right)$ shift to higher frequencies and the $\mathrm{E}^{\prime}\left(\mathrm{E}_{\mathrm{g}}\right)$ displace to lower frequencies (see table 1 ), a behavior that has been reported experimentally by different authors in $\mathrm{WSe}_{2}$ and other STMDs ${ }^{15,19,21-23,29}$. Experimentally, these modes in the bulk 3-D crystals are associated with the $A_{1 g}$ (located at $251 \mathrm{~cm}^{-1}$ ) and the $E_{2 g}$ (around $247 \mathrm{~cm}^{-1}$ ) irreducible representations of the $D_{6 h}$ point group, exhibiting inversion symmetry (see table 1$)^{22,30}$.

From the Raman spectra we noticed that with the laser line of $514.5 \mathrm{~nm}$ a new peak located at $310.4 \mathrm{~cm}^{-1}$ appears for the bilayer, tri-layer, tetra-layer and penta-layer (see figure $2 \mathrm{a}-\mathrm{d}$ ). Other authors have identified this peak at $308 \mathrm{~cm}^{-121}, 309 \mathrm{~cm}^{-122}$ and $310 \mathrm{~cm}^{-119}$. One report assigned this peak to a combination of shear modes and the $E_{2 g}$ mode $e^{21}$, and another publication related this peak to the inactive $\mathrm{B}_{2 \mathrm{~g}}$ mode of bulk WSe ${ }_{2}{ }^{22}$. Our calculations on FLSTMD predicted Raman-active modes around $306 \mathrm{~cm}^{-1}$ (See table 1) that are close in frequency to the infrared-active $\mathrm{A}_{2 \mathrm{u}}\left(305 \mathrm{~cm}^{-1}\right)^{31}$ and the Raman-inactive $B_{2 g}$ mode of the bulk phase $\left(301 \mathrm{~cm}^{-1}\right)^{30}$. These new
Raman-active modes involve the vibrations of the chalcogen and the transition metal atoms, belonging to the $\mathrm{A}^{\prime}{ }_{1}$ or $\mathrm{A}_{1 \mathrm{~g}}$ irreducible representations (for odd and even number of layers, respectively), and do not appear in the monolayer. We propose to label these new modes $\mathrm{A}^{\prime}{ }_{1}{ }^{2}$ and $\mathrm{A}_{1 \mathrm{~g}}{ }^{2}$ (See table 1 and figure 3). For the bi-layer $\left(306.97 \mathrm{~cm}^{-1}\right)$ and tri-layer $\left(306.57 \mathrm{~cm}^{-1}\right)$, only one Raman-active mode appears around this frequency, but for the tetra-layer $\left(305.57 \mathrm{~cm}^{-1}\right.$ and $\left.306.62 \mathrm{~cm}^{-1}\right)$ and penta-layer $\left(305.29 \mathrm{~cm}^{-1}\right.$ and $306.84 \mathrm{~cm}^{-1}$ ), two modes appear that are very close in frequency, corresponding to exterior and interior layer vibrations (See table 1 and figure 4).

In order to experimentally demonstrate that these first order high frequency modes belong to vibrations perpendicular to the lattice plane, parallel and cross polarized Raman spectra were acquired using a $514.5 \mathrm{~nm}$ laser, confirming that both the $\mathrm{A}_{1 \mathrm{~g}}$ and $\mathrm{A}^{\prime}{ }_{1}$ signals for systems with different number of layers vanish completely in the cross-polarized Raman configuration ( $\mathrm{VH}$ ), as expected by group theory (See figure 3 ). In figure 3 it is also shown a signal around $260 \mathrm{~cm}^{-1}$ for monolayer, bilayer and trilayer which under no strain could be regarded as a second order peak caused by a double resonance effect involving the LA(M) phonon located around $130 \mathrm{~cm}^{-1}$ (See figure 3d) which will produce a peak circa $260 \mathrm{~cm}^{-1}$; double resonance effects involving the LA(M) phonon have already been reported in $\mathrm{WS}_{2}{ }^{15}$.

Interestingly, these new modes have also been reported experimentally in $\mathrm{MoSe}_{2}$ at ca. $353 \mathrm{~cm}^{-122}$, and appear in our calculations between $345.54 \mathrm{~cm}^{-1}$ and $348.07 \mathrm{~cm}^{-1}$, depending on the number of layers (See table 1). For few-layered $\mathrm{MoS}_{2}$ and $\mathrm{WS}_{2}$, the high frequency out of plane modes, according to DFPT, are around $463 \mathrm{~cm}^{-1}$ and $437 \mathrm{~cm}^{-1}$, respectively. Unfortunately, these modes have not been properly identified experimentally for several reasons. First, for $\mathrm{MoS}_{2}$ the mode at $464 \mathrm{~cm}^{-1}$ is close to the $2 \mathrm{LA}(\mathrm{M})$ mode located at $455 \mathrm{~cm}^{-1}$ and it is often confused with it, and sometimes assigned to the Raman inactive mode in the bulk crystal, the $\mathrm{A}_{2 \mathrm{u}}$ mode, but no further discussion has been made in the literature $\mathrm{e}^{23,32}$. Second, for few layered $\mathrm{WS}_{2}$ the mode centered at $437 \mathrm{~cm}^{-1}$ exhibits a very small intensity, probably due to the small polarizability of this mode (the components of the Raman tensor are very small), thus making it difficult to identify this mode experimentally.

In addition, our calculations in FLSTMDs reveal Raman-active splitting modes, both out of plane and in-plane; this can be seen for instance from the phonon dispersions of $\mathrm{WSe}_{2}$ at the $\Gamma$ point (see figure $1 \mathrm{~S}$ in supplementary information). While mono-layered and bi-layered $\mathrm{WSe}_{2}$ exhibit only one perpendicular mode at $250.23 \mathrm{~cm}^{-1}$ and $250.83 \mathrm{~cm}^{-1}$, respectively, the tri-layer $\left(249.17 \mathrm{~cm}^{-1}\right.$ and $\left.251.23 \mathrm{~cm}^{-1}\right)$ and tetra-layer $\left(249.58 \mathrm{~cm}^{-1}\right.$ and $\left.251.3 \mathrm{~cm}^{-1}\right)$ possess two branches, and the penta-layer reveals three $\left(248.83 \mathrm{~cm}^{-1}, 250.15 \mathrm{~cm}^{-1}\right.$ and $251.21 \mathrm{~cm}^{-1}$ ) (See table 1 and figure 4). Recently, these three out of plane modes have been reported experimentally in five layered $\mathrm{MoSe}_{2}$ at $238.03 \mathrm{~cm}^{-1}$, $240.12 \mathrm{~cm}^{-1}$ and $242.04 \mathrm{~cm}^{-122}$; in our calculations, these modes appear at $238.42 \mathrm{~cm}^{-1}, 240.02 \mathrm{~cm}^{-1}$ and $241.4 \mathrm{~cm}^{-1}$ and are in very good agreement with the experimental data (See table 1). The main reason why we cannot experimentally resolve them in $\mathrm{WSe}_{2}$ is because the in-plane modes are very close in frequency to the perpendicular modes, thus making this task very difficult and dependent 

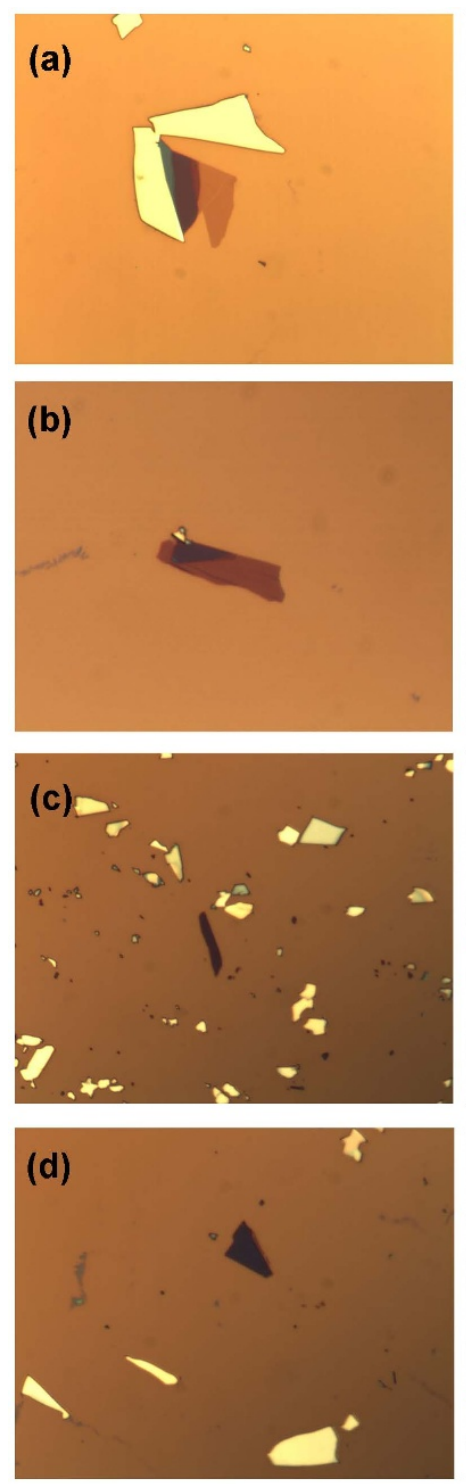
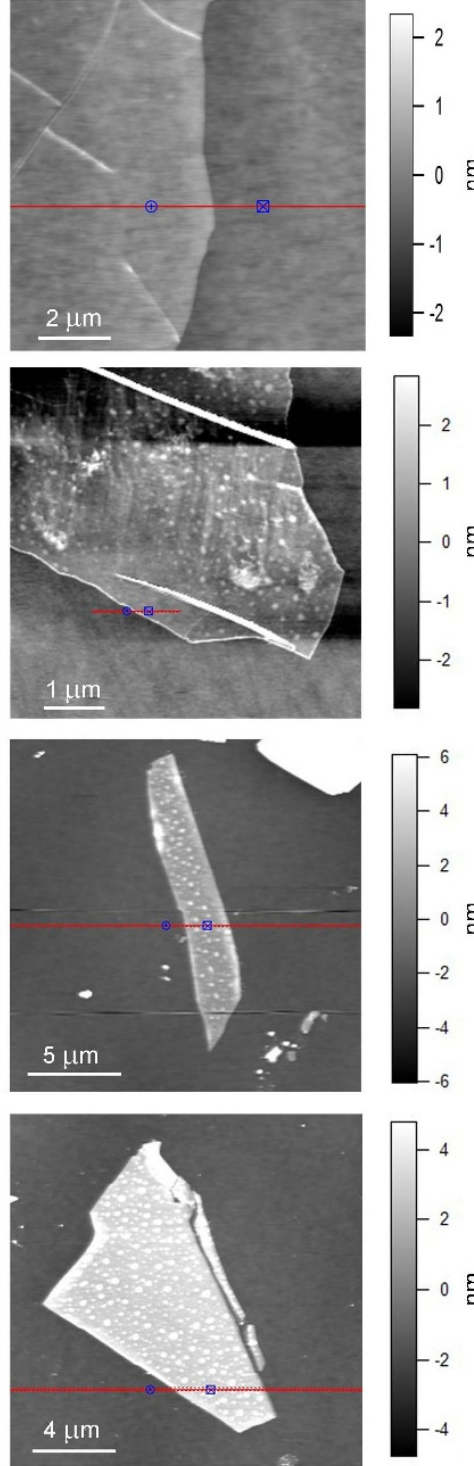
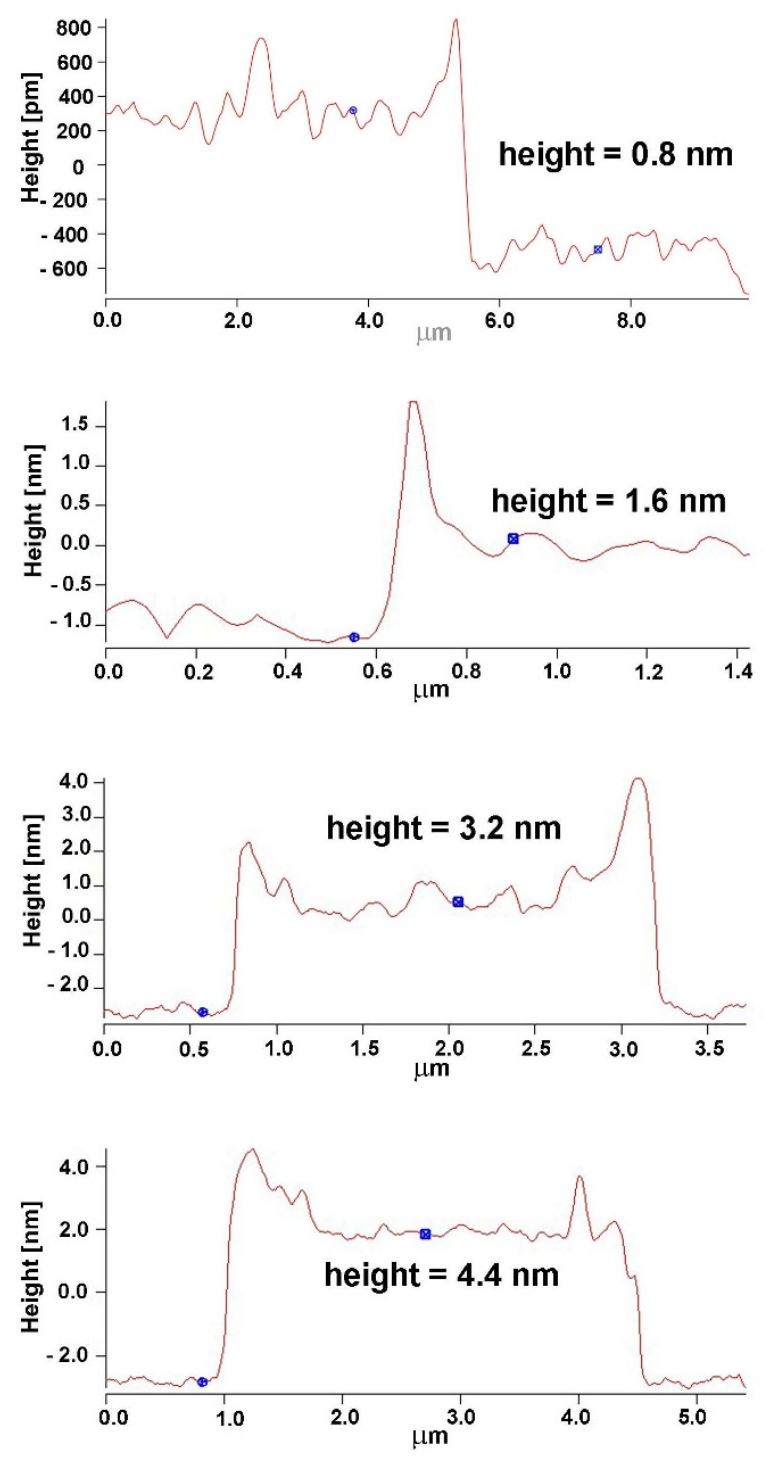

Figure 1 | Optical and atomic force microscope images of exfoliated WSe ${ }_{2}$ crystals a) Monolayer; b) Bilayer; c)Tetra-layer; d) Penta-layer.

on the resolution of the instrument. In other FLSTMDs, such as $\mathrm{WS}_{2}$ and $\mathrm{MoS}_{2}$, the in-plane and out of plane signals are separated by several wave numbers, around $60 \mathrm{~cm}^{-1}$ for $\mathrm{WS}_{2}$ and $20 \mathrm{~cm}^{-1}$ for $\mathrm{MoS}_{2}$, thus making possible to identify the splitting when having a high resolution instrument working at low temperatures. For $\mathrm{WS}_{2}$, our calculations depict that there should be two modes for both the tri-layer and tetra-layer between $416.87 \mathrm{~cm}^{-1}$ and $419.81 \mathrm{~cm}^{-1}$ (see table 1), and for the penta-layer there should be three modes between $416.73 \mathrm{~cm}^{-1}$ and $420.14 \mathrm{~cm}^{-1}$. In the case of the $\mathrm{MoS}_{2}$ tri-layer and tetra-layer, the two splitting modes appear between $400 \mathrm{~cm}^{-1}$ and $403 \mathrm{~cm}^{-1}$, and for the penta-layer, three modes appear between $399.73 \mathrm{~cm}^{-1}$ and $403.44 \mathrm{~cm}^{-1}$ (See table 1). On the other hand, it might be interesting to try finding the splitting in other $2 \mathrm{D}$ materials.

Our calculations indicate that the splitting of $\mathrm{A}^{\prime}{ }_{1}\left(\mathrm{~A}_{\mathrm{gg}}\right)$, starting from $\mathrm{L}=3$, involves two main types of mode families: One, in which all the Se or S atoms compress or expand at the same time, thus shifting to higher frequencies as the number of layers grows (reaching the maximum frequency in bulk), and another, in which the chalcogen vibrations in each layer exhibit opposite directions in an alternative way, resulting in lower frequencies (See table 1 and figure 4).

It is also noteworthy that the new in-plane modes can differentiate between interior and exterior layers, and belong to the $\mathrm{E}_{\mathrm{g}}$ irreducible representation for even numbers of layers, and to the $\mathrm{E}^{\prime}$ and $\mathrm{E}^{\prime \prime}$ for odd numbers of layers. In $\mathrm{WSe}_{2}$ the in plane modes are around $176 \mathrm{~cm}^{-1}$ and $248 \mathrm{~cm}^{-1}$ agreeing with the calculations presented by Luo and co-workers for $\mathrm{L}=2$ to $\mathrm{L}=4^{19}$ (see table 1 ). In our Raman spectra, despite using different laser lines, it is difficult to observe a signal around $176 \mathrm{~cm}^{-1}$ due to the small intensity of these modes, however, around $248 \mathrm{~cm}^{-1}$ we observe the signal of these modes which is mixed with the out of plane modes (see figure 2). For the case of $\mathrm{MoSe}_{2}$ the in-plane modes are located circa $167 \mathrm{~cm}^{-1}$ and $283 \mathrm{~cm}^{-1}$, in WS 2 at about $298 \mathrm{~cm}^{-1}$ and $357 \mathrm{~cm}^{-1}$, and in $\mathrm{MoS}_{2}$ are nearby $282 \mathrm{~cm}^{-1}$ and $380 \mathrm{~cm}^{-1}$ (to differentiate between these in plane modes a super index has been used in table 1). For example, in $\mathrm{WSe}_{2}$, two in-plane modes appear for the tri-layer $\left(247.83 \mathrm{~cm}^{-1}\right.$ and $\left.248.33 \mathrm{~cm}^{-1}\right)$ and tetra-layer $\left(247.66 \mathrm{~cm}^{-1}\right.$ and $\left.248.35 \mathrm{~cm}^{-1}\right)$, and three modes appeared for the penta-layer $\left(247.55 \mathrm{~cm}^{-1}, 247.65 \mathrm{~cm}^{-1}\right.$ and $248.55 \mathrm{~cm}^{-1}$. See figure 4). Further, it is worth noticing that Raman-active $\mathrm{E}^{\prime \prime}$ modes are also active for the tri-layer at $248.32 \mathrm{~cm}^{-1}$ and the penta-layer at $248.54 \mathrm{~cm}^{-1}$, and only the exterior layers are involved (See figure 4). In addition, the $E^{\prime}$ in-plane mode has its maximum frequency value for the monolayer, and when more layers are added, a splitting takes place in which the interior layers (bulk-like) exhibit lower frequencies than the exterior ones (surface layers). Therefore, when reaching the bulk phase (no 
(a)

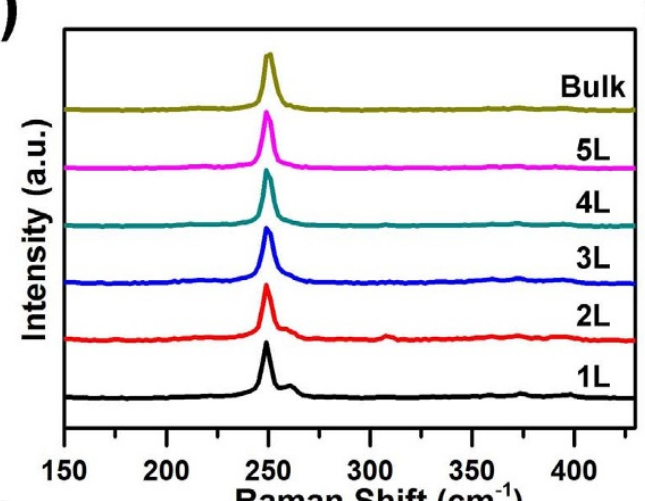

(c)

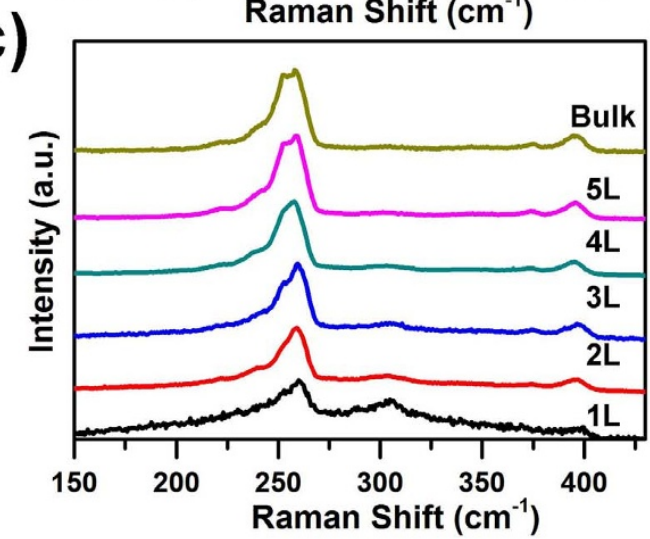

(b)

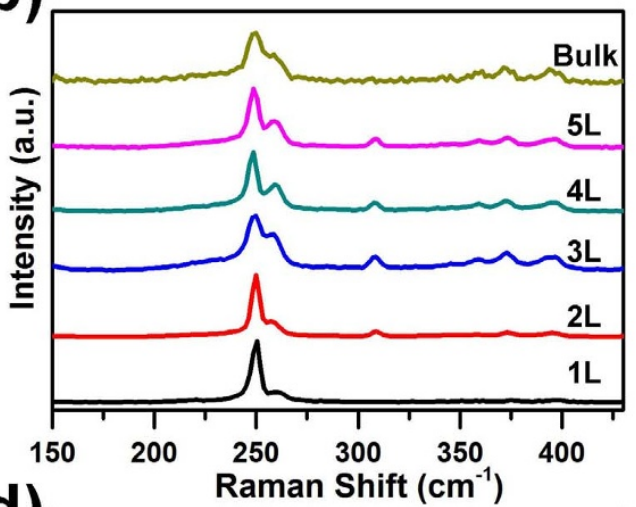

(d)

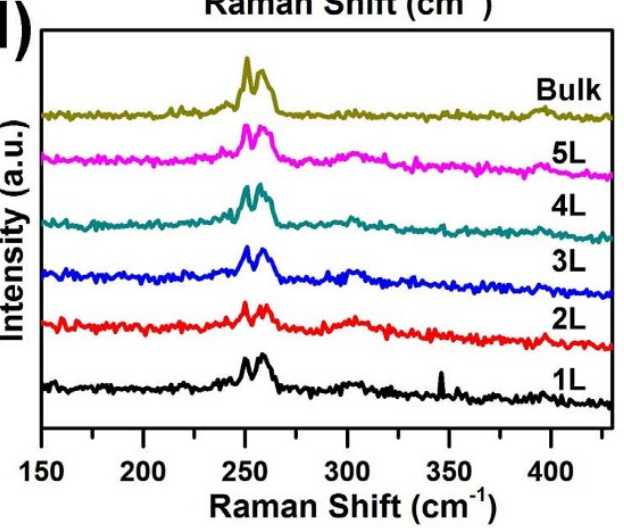

\section{Energy (eV)}

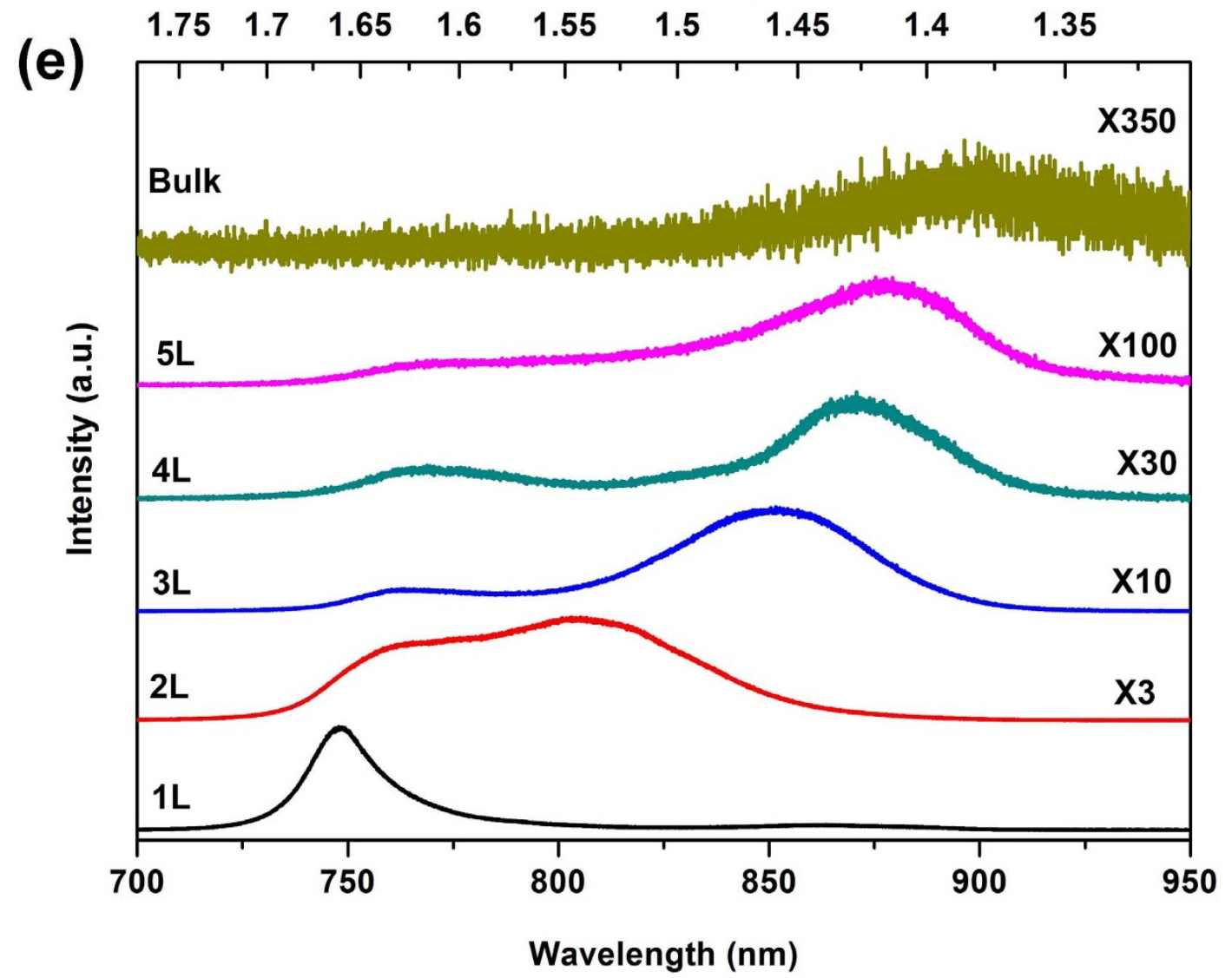

Figure $2 \mid$ Raman spectra of few layered WSe $e_{2}$ with different excitation wavelengths; (a) $488 \mathrm{~nm}$; (b) $514.5 \mathrm{~nm}$; (c) $633 \mathrm{~nm}$; (d) $647 \mathrm{~nm}$; (e) Photoluminescence (PL) spectra obtained with the $488 \mathrm{~nm}$. 
(a)
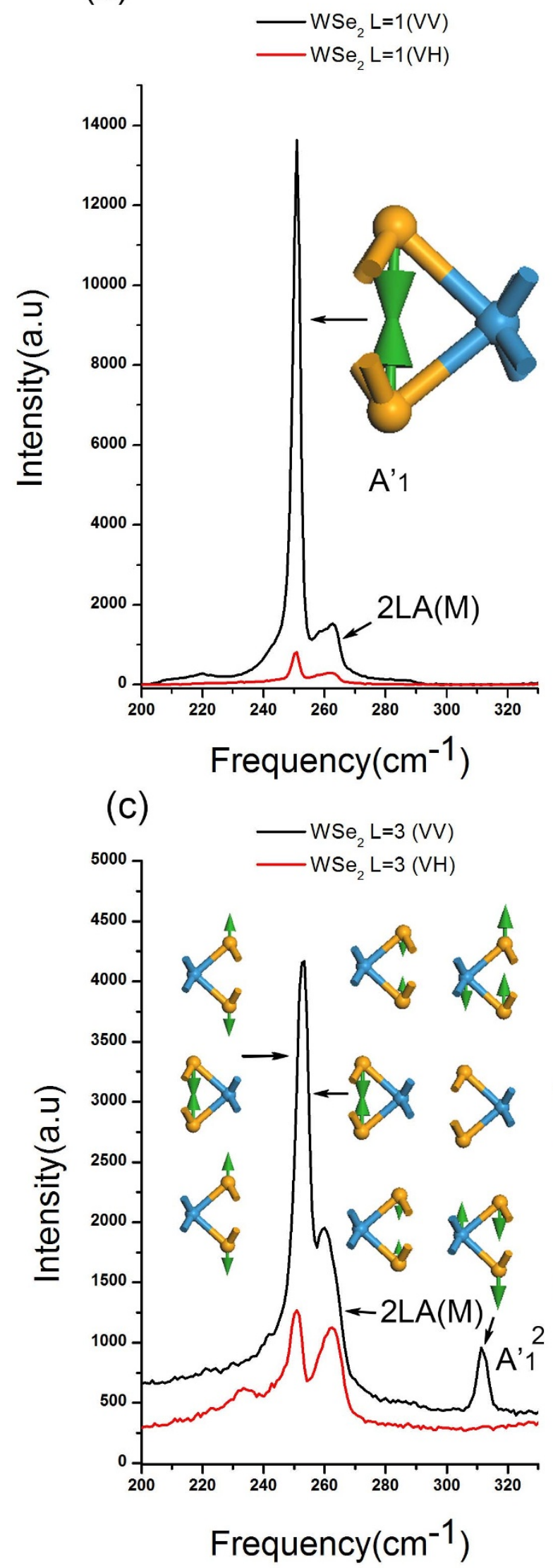

(b)

$$
\begin{aligned}
& -\mathrm{WSe}_{2} \mathrm{~L}=2(\mathrm{VV}) \\
& -\mathrm{WSe}_{2} \mathrm{~L}=2(\mathrm{VH})
\end{aligned}
$$

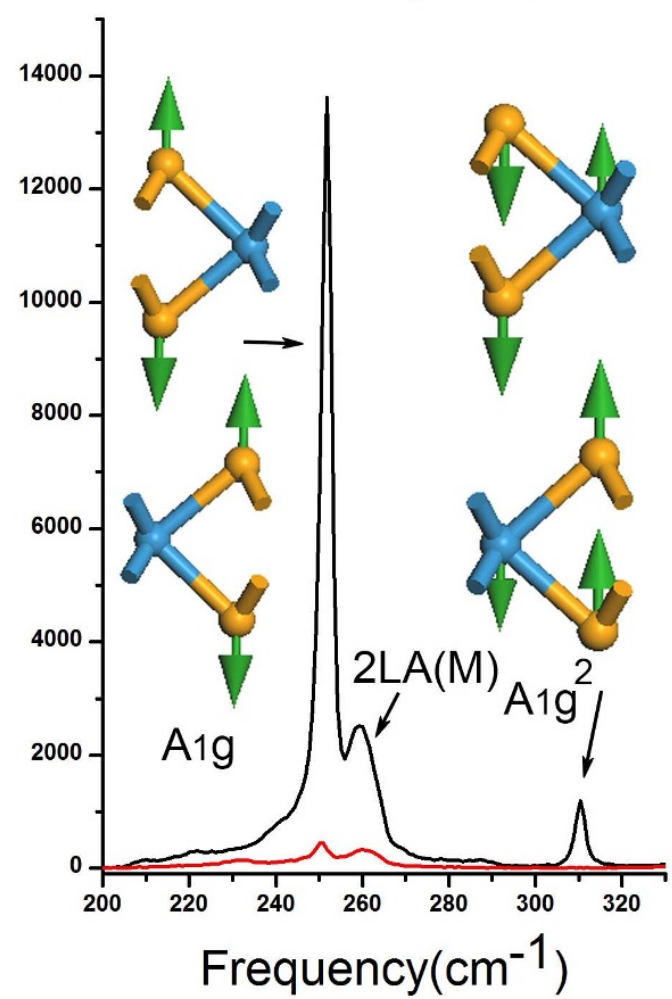

(d)

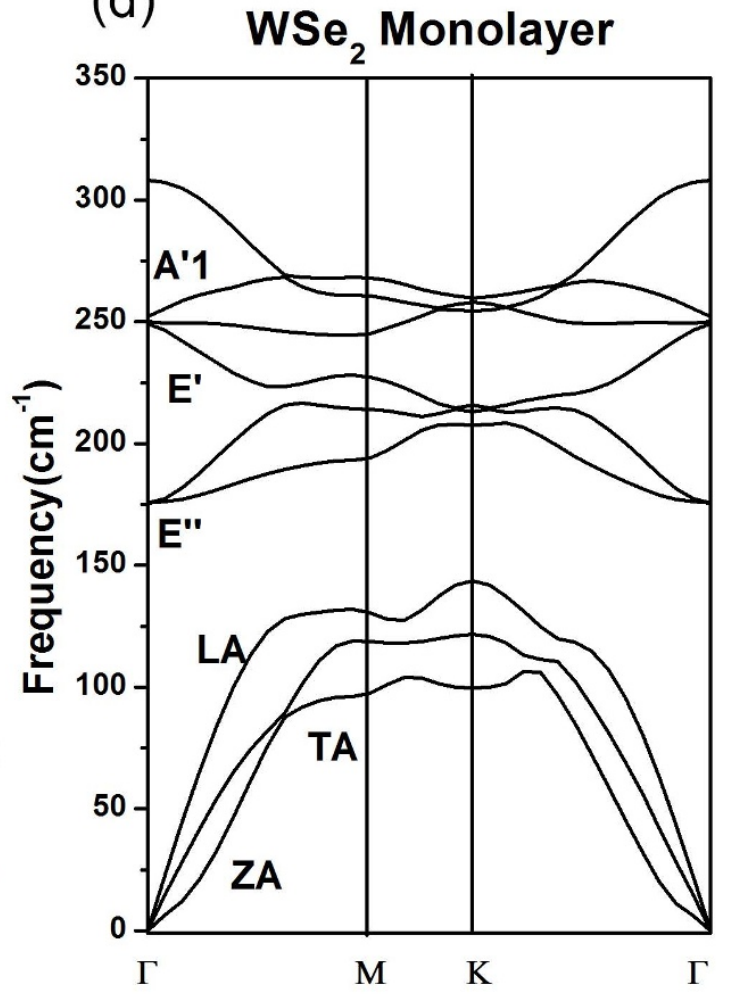

Figure 3 Experimental Raman spectra of $\mathrm{WSe}_{2}$ taken with a $514 \mathrm{~nm}$ laser under conventional geometry (VV) and cross polarization (VH). (a) Monolayer $(\mathrm{L}=1)$. (b) Bilayer $(\mathrm{L}=2)$. (c) Trilayer $(\mathrm{L}=3)$. Insets show models of the out of plane vibrational modes present in each case. (d) Calculated DFPT phonon dispersion of the $\mathrm{WSe}_{2}$ monolayer. 

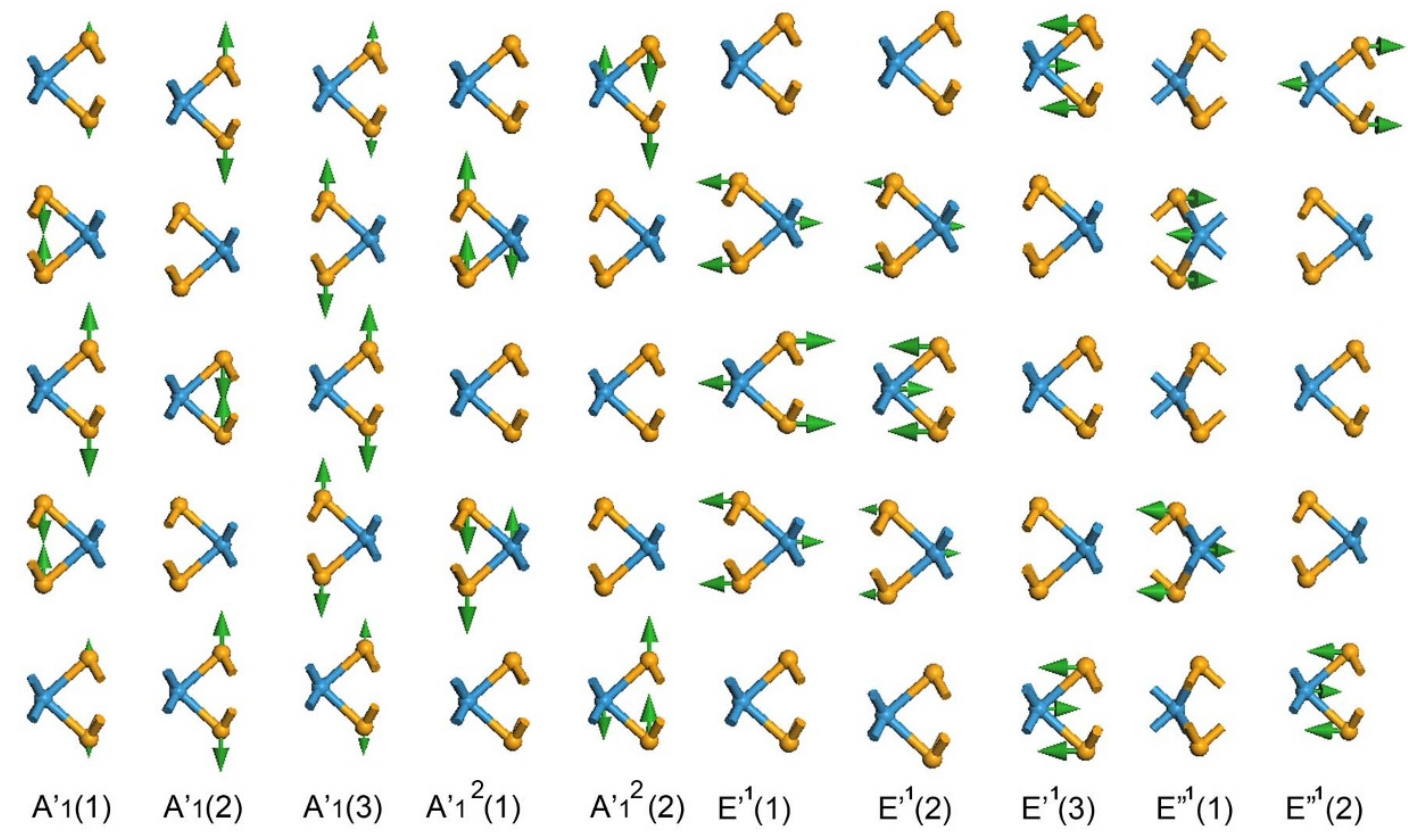

K
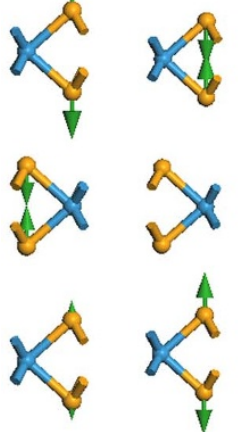

A'1(2)

$A^{\prime} 1(3)$

$A^{\prime}(1)$

$A^{\prime} 1$ (2) $E^{, 1}(1)$
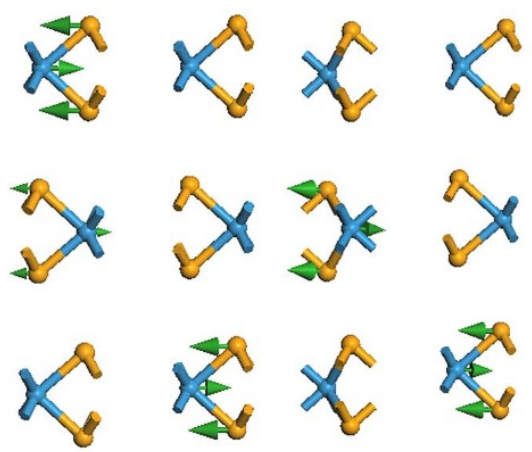

$\mathrm{E}^{, 1}(2)$

$E^{\prime 1}(3)$
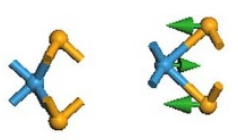

$E^{\prime \prime}(1)$

E" (2)

Figure $4 \mid$ Models showing the vibrations of $\mathrm{A}^{\prime}{ }_{1}, \mathrm{E}^{\prime}$ and $\mathrm{E}^{\prime \prime}$ Raman-active modes for $\mathrm{L}=5 \mathrm{WSe}_{2} \cdot \mathrm{A}^{\prime}{ }_{1}(1)$ at $248.83 \mathrm{~cm}^{-1} ; \mathrm{A}^{\prime}{ }_{1}(2)$ at $250.15 \mathrm{~cm}^{-1} ; \mathrm{A}^{\prime}{ }_{1}(3)$ at $251.21 \mathrm{~cm}^{-1} ; \mathrm{A}^{\prime}{ }_{1}{ }^{2}(1)$ at $305.29 \mathrm{~cm}^{-1} ; \mathrm{A}^{\prime}{ }_{1}{ }^{2}(2)$ at $306.84 \mathrm{~cm}^{-1} ; \mathrm{E}^{\prime 1}(1)$ at $247.55 \mathrm{~cm}^{-1} ; \mathrm{E}^{\prime 1}(2)$ at $247.65 \mathrm{~cm}^{-1} ; \mathrm{E}^{\prime 1}(3)$ at $248.55 \mathrm{~cm}^{-1}$; $\mathrm{E}^{\prime \prime 1}(1)$ at $247.59 \mathrm{~cm}^{-1}$ and $\mathrm{E}^{\prime \prime 1}(2)$ at $248.54 \mathrm{~cm}^{-1}$.

exterior layers), the $\mathrm{E}_{1 \mathrm{~g}}$ mode preserves the lower frequency values of the interior layers, thus explaining the experimentally observed shift to lower frequencies as the number of layers is increased (See figure 4). All the $\mathrm{E}^{\prime}$ modes in odd numbers of layers are also infraredactive (See table 1), while for even numbers of layers the $E_{1 \mathrm{~g}}$ modes, as expected from the principle of mutual exclusion, are not infraredactive since they are symmmetric with respect to inversion. The calculated phonon dispersions of $\mathrm{WSe}_{2}$ for $\mathrm{L}=1$ to $\mathrm{L}=5$ and bulk are shown in figure $S 1$ (supplementary information) where the new modes appear as new branches at the $\Gamma$ point. These results can be extended to other FLSTMDs with trigonal prismatic layers (See table 1).

We also expect that the new modes mentioned above will appear in hetero-bilayered systems formed by stacking different layers of STMDs. For example, for a bilayered system made of one layer of $\mathrm{WSe}_{2}$ and another layer of $\mathrm{WS}_{2}$, our calculations demonstrate that, besides the $A_{1}\left(C_{3 v}\right.$ symmetry) vibrational modes at $251.7 \mathrm{~cm}^{-1}$ for $\mathrm{WSe}_{2}$ and at $415.4 \mathrm{~cm}^{-1}$ for $\mathrm{WS}_{2}$, two other perpendicular Ramanactive modes appear at different frequencies, one at $311.1 \mathrm{~cm}^{-1}$ for $\mathrm{WSe}_{2}$ and another at $427.9 \mathrm{~cm}^{-1}$ for $\mathrm{WS}_{2}$. Note that these modes are close in frequency to those present in the pure bilayered systems. However, the shift here is mainly due to an induced strain causing the $\mathrm{WS}_{2}$ to expand and the $\mathrm{WSe}_{2}$ to contract to preserve an energy local minimum ${ }^{33}$. Since our calculations consider free standing layers of STMDs, the effect of the substrate has not been considered and could affect mainly the layer to which it has interaction depending on the particular substrate: Several experimental works, including ours, use $\mathrm{SiO}_{2} / \mathrm{Si}$ substrates ${ }^{16,21-23,29,34}$, and the Raman modes exhibit little change from those calculated here and by other authors when no strain is considered ${ }^{18,19,35}$, indicating a weak interaction with the substrate. In addition, the photoluminescence results (See figure 2) show a clear signal at $750 \mathrm{~nm}$ which corresponds to the direct optical band gap of monolayer $\mathrm{WSe}_{2}$, thus no evident strain from the substrate or from the sample itself is detected since strain would alter the optical band gap ${ }^{35-37}$. Nevertheless, a careful study with different substrates is required to address the role of a particular substrate with FLSTMDs.

In summary, we have addressed Raman spectroscopic modes of FLSTMDs by measuring directly few layered exfoliated $\mathrm{WSe}_{2}$ samples, and confirming our observations by performing DFPT first principles calculations. We find that there are novel, out of plane and in plane, Raman-active modes that are not present in the monolayer or in the bulk, and are capable of differentiating between the vibrations of the outer and interior layers. Our results also explain recent observations reported for few layered $\mathrm{WSe}_{2}, \mathrm{MoSe}_{2}$ and $\mathrm{MoS}_{2}$. These new modes reported here will be important when characterizing fewlayered systems as well as to identify monolayers. In addition, the new modes might play an important role in double resonance Raman processes in FLSTMDs.

\section{Methods}

In order to study and understand the origin of the main features observed in Raman spectra of FLSTMDs, ab-initio density functional theory (DFT) and density functional perturbation theory (DFPT) calculations were carried out in monolayers and few-layered systems of WSe $\mathrm{WoSe}_{2}, \mathrm{MoS}_{2}$ and $\mathrm{WS}_{2}$ (from $\mathrm{L}=1$ to $\mathrm{L}=5$, and also the bulk), as implemented in the plane wave code CASTEP ${ }^{38}$ in the Materials Studio package. Trigonal unit cells were considered under the local density approximation (LDA) using the Ceperly-Alder-Perdew and Zunger (CA-PZ) functional ${ }^{39,40}$ with $9 \times$ $9 \times 3$ Monkhorst-Pack K-points and a plane waves cut off of $500 \mathrm{eV}$ with a normconserving pseudopotential. All the structures were relaxed, including the cells, until the forces became smaller than $0.01 \mathrm{eV} / \AA$ and with self- consistent energy tolerances less than $5 \times 10^{-7} \mathrm{eV} /$ atom. A vacuum of $16 \AA$ between the layers was considered. The electronic structure results obtained are in agreement with those reported by other groups within the LDA formalism ${ }^{41,42}$. In order to provide a reasonable description of the van der Waals interaction, we have considered a dispersion correction for both LDA (LDA-D) ${ }^{43}$ and for general gradient approximation (GGAPW91 $)^{44}$ finding that LDA provides a better description for the interlayer distance than LDA-D and corrected GGA-PW91, as described in a previous publication ${ }^{33}$. The phonon dispersions and Raman scattering modes were calculated with the above parameters, but extending the plane wave cut off to $720 \mathrm{eV}$ with DFPT using the linear response methodology for insulators ${ }^{45}$.

The few layered $\mathrm{WSe}_{2}$ samples were obtained by mechanical exfoliation of $\mathrm{WSe}_{2}$ crystals produced using the chemical vapor transport method with iodine as transport agent $^{24-26}$. First, $\mathrm{WSe}_{2}$ powder was synthesized by heating a mixture containing stoichiometric amounts of tungsten (Acros Organics 99.9\%) and selenium (Acros Organics $99.5+\%$ ) at $1000^{\circ} \mathrm{C}$ for 3 days in an evacuated and sealed quartz ampoule (10 mm ID, $12 \mathrm{~mm}$ OD, $150 \mathrm{~mm}$ length). The mixture was slowly heated from room temperature to $1000^{\circ} \mathrm{C}$ for 12 hours, in order to avoid any explosion due to the strong exothermic reaction. The $\mathrm{WSe}_{2}$ crystals were grown from that synthesized powder using chemical vapor transport with iodine (Sigma-Aldrich, 99.8+\%) as the transport gas at $1.5 \mathrm{mg} / \mathrm{cm}^{3}$. The experiments ran for 10 days in an evacuated and sealed quartz ampoule (10 mm ID, $12 \mathrm{~mm} \mathrm{OD,} 100 \mathrm{~mm}$ length). The source and growth zones were kept at $950^{\circ} \mathrm{C}$ and $816^{\circ} \mathrm{C}$, respectively. The resulted crystals were washed with 
hexane and dried in vacuo to remove any residual iodine. Both $\mathrm{WSe}_{2}$ powder and crystals were analyzed using XRD, which confirmed that both $\mathrm{WSe}_{2}$ powders and crystals were pure $2 \mathrm{H}$ phase (trigonal prismatic). The micro-Raman measurements were performed in a triple monochromator spectrometer (DILOR XY) equipped with an $\mathrm{Ar} / \mathrm{Kr}$ laser and in a Renishaw inVia confocal microscope-based Raman spectrometer using the $488 \mathrm{~nm}, 514.5 \mathrm{~nm}$ and $633 \mathrm{~nm}$ and $647 \mathrm{~nm}$ excitation wavelengths. A back scattering geometry at room temperature was used and the laser power was kept below $0.6 \mathrm{~mW}$ to avoid damage on the samples. The laser spot size using the $100 \times$ objective was about $4 \mu \mathrm{m}^{2}$, always smaller that the dimensions of the flakes. A $\lambda / 2$ polarizer was used to achieve the VV and VH configurations. The accumulations times varies between 5 and 10 minutes depending on the sample and the excitation wavelength.

1. Hwang, W. S. et al. Transistors with chemically synthesized layered semiconductor $\mathrm{WS}_{2}$ exhibiting $10^{5}$ room temperature modulation and ambipolar behavior. Appl. Phys. Lett. 101, doi:013107 10.1063/1.4732522 (2012).

2. Wang, Q. A., Kalantar-Zadeh, K., Kis, A., Coleman, J. N. \& Strano, M. S Electronics and optoelectronics of two-dimensional transition metal dichalcogenides. Nat. Nanotechnol. 7, 699-712, doi:doi:10.1038/nnano.2012.193 (2012).

3. Wang, H. et al. Integrated Circuits Based on Bilayer $\mathrm{MoS}_{2}$ Transistors. Nano Lett. 12, 4674-4680, doi:10.1021/nl302015v (2012).

4. Zhang, Y. J., Ye, J. T., Matsuhashi, Y. \& Iwasa, Y. Ambipolar $\mathrm{MoS}_{2}$ Thin Flake Transistors. Nano Lett. 12, 1136-1140, doi:10.1021/nl2021575 (2012).

5. Fang, H. et al. High-Performance Single Layered WSe2 p-FETs with Chemically Doped Contacts. Nano Lett. 12, 3788-3792, doi:10.1021/nl301702r (2012)

6. Liu, W. et al. Role of Metal Contacts in Designing High-Performance Monolayer n-Type WSe2 Field Effect Transistors. Nano Lett. 13, 1983-1990, doi:10.1021/ nl304777e (2013).

7. Das, S., Chen, H.-Y., Penumatcha, A. V. \& Appenzeller, J. High Performance Multilayer MoS2 Transistors with Scandium Contacts. Nano Lett. 13, 100-105, doi:10.1021/nl303583v (2012).

8. Mak, K. F., Lee, C., Hone, J., Shan, J. \& Heinz, T. F. Atomically Thin $\mathrm{MoS}_{2}$ : A New Direct-Gap Semiconductor. Phys. Rev. Lett. 105, doi:10.1103/ PhysRevLett.105.136805 (2010).

9. Splendiani, A. et al. Emerging Photoluminescence in Monolayer $\mathrm{MoS}_{2}$. Nano Lett. 10, 1271-1275, doi:10.1021/nl903868w (2010).

10. Korn, T., Heydrich, S., Hirmer, M., Schmutzler, J. \& Schuller, C. Low-temperature photocarrier dynamics in monolayer $\mathrm{MoS}_{2}$. Appl. Phys. Lett. 99, doi:10.1063/ 1.3636402 (2011)

11. Eda, G. et al. Photoluminescence from Chemically Exfoliated $\mathrm{MoS}_{2}$. Nano Lett. 11, 5111-5116, doi:10.1021/nl201874w (2011).

12. Mak, K. F., He, K. L., Shan, J. \& Heinz, T. F. Control of valley polarization in monolayer $\mathrm{MoS}_{2}$ by optical helicity. Nat. Nanotechnol. 7, 494-498, doi:10.1038/ nnano.2012.96 (2012).

13. Zeng, H. L., Dai, J. F., Yao, W., Xiao, D. \& Cui, X. D. Valley polarization in $\mathrm{MoS}_{2}$ monolayers by optical pumping. Nat. Nanotechnol. 7, 490-493, doi:10.1038/ nnano.2012.95 (2012).

14. Cao, T. et al. Valley-selective circular dichroism of monolayer molybdenum disulphide. Nat Commun 3, 887, doi:10.1038/ncomms1882 (2012).

15. Gutiérrez, H. R. et al. Extraordinary Room-Temperature Photoluminescence in Triangular WS2 Monolayers. Nano Lett. 13, 3447-3454, doi:10.1021/nl3026357 (2013).

16. Berkdemir, A. et al. Identification of individual and few layers of WS2 using Raman Spectroscopy. Sci. Rep. 3, doi:10.1038/srep01755 (2013).

17. Sun, L. et al. Spin-Orbit Splitting in Single-Layer $\mathrm{MoS}_{2}$ Revealed by Triply Resonant Raman Scattering. Phys. Rev. Lett. 111, 126801, doi:10.1103/ PhysRevLett.111.126801 (2013)

18. Sahin, H. et al. Anomalous Raman spectra and thickness-dependent electronic properties of WSe 2 . Phys. Rev. B 87, 165409, doi:10.1103/PhysRevB.87.165409 (2013).

19. Luo, X. et al. Effects of lower symmetry and dimensionality on Raman spectra in two-dimensional WSe. Phys. Rev. B 88, 195313, doi:10.1103/ PhysRevB.88.195313 (2013).

20. Verble, J. L. \& Wieting, T. J. Lattice Mode Degeneracy in $\mathrm{MoS}_{2}$ and Other Layer Compounds. Phys. Rev. Lett. 25, 362-365, doi:10.1103/PhysRevLett.25.362 (1970).

21. Zhao, W. et al. Lattice dynamics in mono- and few-layer sheets of $\mathrm{WS}_{2}$ and $\mathrm{WSe}_{2}$. Nanoscale, doi:10.1039/C3NR03052K (2013).

22. Tonndorf, P. et al. Photoluminescence emission and Raman response of monolayer $\mathrm{MoS}_{2}, \mathrm{MoSe}_{2}$, and WSe 2 . Opt. Express 21, 4908-4916 (2013).

23. Zhang, X. et al. Raman spectroscopy of shear and layer breathing modes in multilayer $\mathrm{MoS}_{2}$. Phys. Rev. B 87, 115413, doi:10.1103/PhysRevB.87.115413 (2013).

24. Schäfer, H. Chemical Transport Reactions. (Academic Press Inc., New York, 1964)

25. Schäfer, H., Grofe, T. \& Trenkel, M. Chemical Transport of Molybdenum and Tungsten and of Their Dioxides and Sulfides. J. Solid State Chem. 8, 14-28, doi:10.1016/0022-4596(73)90014-5 (1973).

26. Lieth, R. M. A. Preparation and Crystal Growth of Materials with Layered Structures. Vol. 1 (Springer Netherlands, 1977).
27. Kumar, N. et al. Second harmonic microscopy of monolayer $\mathrm{MoS}_{2}$. Phys. Rev. B 87, 161403, doi:10.1103/PhysRevB.87.161403 (2013).

28. Malard, L. M., Alencar, T. V., Barboza, A. P. M., Mak, K. F. \& de Paula, A. M. Observation of intense second harmonic generation from $\mathrm{MoS}_{2}$ atomic crystals. Phys. Rev. B 87, doi:10.1103/PhysRevB.87.201401 (2013).

29. Lee, C. et al. Anomalous Lattice Vibrations of Single- and Few-Layer MoS2. ACS Nano 4, 2695-2700, doi:10.1021/nn1003937 (2010).

30. Sekine, T., Izumi, M., Nakashizu, T., Uchinokura, K. \& Matsuura, E. RamanScattering and Ifrared Reflectance in $2 \mathrm{H}-\mathrm{MoSe}_{2}$. J. Phys. Soc. Jpn. 49, 1069-1077, doi:10.1143/jpsj.49.1069 (1980).

31. Lucovsky, G., White, R. M., Benda, J. A. \& Revelli, J. F. Infrared-Reflectance Spectra of Layered Group-IV and Group-VI Transition-Metal Dichalcogenides. Phys. Rev. B 7, 3859-3870 (1973).

32. Chakraborty, B., Matte, H. S. S. R., Sood, A. K. \& Rao, C. N. R. Layer-dependent resonant Raman scattering of a few layer MoS2. J. Raman Spectrosc. 44, 92-96, doi:10.1002/jrs.4147 (2013).

33. Terrones, H., Lopez-Urias, F. \& Terrones, M. Novel hetero-layered materials with tunable direct band gaps by sandwiching different metal disulfides and diselenides. Sci. Rep. 3, doi:10.1038/srep01549 (2013).

34. Tongay, S. et al. Thermally Driven Crossover from Indirect toward Direct Bandgap in 2D Semiconductors: $\mathrm{MoSe}_{2}$ versus $\mathrm{MoS}_{2}$. Nano Lett. 12, 5576-5580, doi:10.1021/nl302584w (2012).

35. Shi, H. L., Pan, H., Zhang, Y. W. \& Yakobson, B. I. Quasiparticle band structures and optical properties of strained monolayer MoS2 and WS2. Phys. Rev. B 87, doi:10.1103/PhysRevB.87.155304 (2013)

36. Feng, J., Qian, X. F., Huang, C. W. \& Li, J. Strain-engineered artificial atom as a broad-spectrum solar energy funnel. Nature Photonics 6, 865-871, doi:10.1038/ nphoton.2012.285 (2012).

37. Conley, H. J. et al. Bandgap Engineering of Strained Monolayer and Bilayer MoS2. Nano Lett. 13, 3626-3630, doi:10.1021/nl4014748 (2013).

38. Clark, S. J. et al. First principles methods using CASTEP. Z. Kristallogr. 220, 567-570, doi:10.1524/zkri.220.5.567.65075 (2005).

39. Ceperley, D. M. \& Alder, B. J. Ground-State of The Electron-Gas by a Stochastic Method. Phys. Rev. Lett. 45, 566-569, doi:10.1103/PhysRevLett.45.566 (1980).

40. Perdew, J. P. \& Zunger, A. Self-Interaction Correction to Density-Functional Approximations for Many-Electron Systems. Phys. Rev. B 23, 5048-5079, doi:10.1103/PhysRevB.23.5048 (1981).

41. Ding, Y. et al. First principles study of structural, vibrational and electronic properties of graphene-like MX2 $(\mathrm{M}=\mathrm{Mo}, \mathrm{Nb}, \mathrm{W}, \mathrm{Ta} ; \mathrm{X}=\mathrm{S}, \mathrm{Se}, \mathrm{Te})$ monolayers. Physica B 406, 2254-2260, doi:10.1016/jphysb.2011.03.044 (2011).

42. Mahatha, S. K., Patel, K. D. \& Menon, K. S. R. Electronic structure investigation of $\mathrm{MoS}_{2}$ and $\mathrm{MoSe}_{2}$ using angle-resolved photoemission spectroscopy and ab initio band structure studies. J. Phys. Condens. Matter 24, doi:10.1088/0953-8984/24/ 47/475504 (2012)

43. Ortmann, F., Bechstedt, F. \& Schmidt, W. G. Semiempirical van der Waals correction to the density functional description of solids and molecular structures. Phys. Rev. B 73, doi:10.1103/PhysRevB.73.205101 (2006).

44. Perdew, J. P. et al. Atoms, Molecules, Solids, And Surfaces-Applications of the Generalized Gradient Approximation for the Exchange and Correlation. Phys. Rev. B 46, 6671-6687, doi:10.1103/PhysRevB.46.6671 (1992).

45. Refson, K., Tulip, P. R. \& Clark, S. J. Variational density-functional perturbation theory for dielectrics and lattice dynamics. Phys. Rev. B 73, doi:10.1103/ PhysRevB.73.155114 (2006).

46. Mead, D. G. \& Irwin, J. C. Long wavelength optic phonons in WSe2. Can. J. Phys 55, 379-382 (1977)

47. Chen, J. M. \& Wang, C. S. Second-order Raman-Spectrum of $\mathrm{MoS}_{2}$. Solid State Commun. 14, 857-860, doi:10.1016/0038-1098(74)90150-1 (1974)

48. Wieting, T. J. \& Verble, J. L. Infrared and Raman Studies of Long-Wavelenght optical Phonons in Hexagonal $\mathrm{MoS}_{2}$. Phys. Rev. B 3, 4286-\&, doi:10.1103/ PhysRevB.3.4286 (1971).

\section{Acknowledgments}

This work was supported by the U.S. Army Research Office MURI grant

W911NF-11-1-0362, by the Materials Simulation Center of the Materials Research Institute (Penn State), the Research Computing and Cyberinfrastructure unit of Information Technology Services and Penn-State Center for Nanoscale Science. M.T. also acknowledges support from the Penn State Center for Nanoscale Science for seed grant on 2-D Layered Materials (DMR-0820404). The authors also acknowledge the Center for 2-Dimensional and Layered Materials at the Pennsylvania State University. M.A.P. and E.D.C. acknowledge the support of INCT Nanocarbono and the Brazilian agencies FAPEMIG and $\mathrm{CNPq}$.

\section{Author contributions}

H.T. performed the calculations and wrote the manuscript. E.D.C., A.L.E., S.F., Z.L. and M.A.P. performed Raman characterization with different lines and discussed the results. J.M.P., D.R., D.S. performed preliminary polarized Raman spectroscopy. N.R.P., S.F. and Z.L. exfoliated the $\mathrm{WSe}_{2}$ crystal samples. D.R., J.M.P., D.S., S.F. and Z.L. performed PL measurements. D.R. and M.A.T.N. worked on the synthesis of the $\mathrm{WSe}_{2}$ crystals. D.R., N.R.P., S.F. and Z.L. performed optical microscopy and AFM measurements. T.E.M. and 
L.B. supervised the synthesis of crystals of $\mathrm{WSe}_{2}$, their exfoliation, optical, Raman and AFM characterization, and discussed the results. M.T. coordinated and revised the theoretical and experimental results, and wrote the manuscript. All authors revised the manuscript.

\section{Additional information}

Supplementary information accompanies this paper at http://www.nature.com/ scientificreports

Competing financial interests: The authors declare no competing financial interests.
How to cite this article: Terrones, H. et al. New First Order Raman-active Modes in Few Layered Transition Metal Dichalcogenides. Sci. Rep. 4, 4215; DOI:10.1038/srep04215 (2014).

(c) (i) $\Theta$ This work is licensed under a Creative Commons AttributionBY NC ND NonCommercial-NoDerivs 3.0 Unported license. To view a copy of this license, visit http://creativecommons.org/licenses/by-nc-nd/3.0 